\title{
La transformación del marketing minorista en la era digital
}

\author{
Hernán Ramón Toniut \\ Universidad Fasta, Mar del Plata, Argentina
}

$\bigotimes$ toniuth@ufasta.edu.ar

望-1] Fecha de recepción: 24/05/2021 - Fecha de aceptación: 28/07/2021

Cómo citar este artículo: Toniut, H. R. (2022). La transformación del marketing minorista en la era digital. Revista Perspectivas de las Ciencias Económicas y Jurídicas. Vol. 12, $\mathrm{N}^{\circ} 1$ (enero-junio). Santa Rosa: FCEyJ (UNLPam); EdUNLPam; pp. 159-182. ISSN 2250-4087, e-ISSN 2445-8566. http://dx.doi.org/10.19137/perspectivas-2022-v12n1a10

Resumen: Las incorporaciones de las nuevas tecnologías de la información y de la comunicación han transformado la forma en la que vivimos, nos informamos, comunicamos, compramos, cómo nos relacionamos con el otro, etc. Estos cambios modifican las áreas funcionales de la organización, siendo el área de marketing una de ellas.

¿Cómo se ha transformado el marketing minorista en la era digital? El artículo, en primer lugar, aborda el concepto, la evolución del marketing en una organización y sus principales variables. En segundo lugar, se desarrolla el concepto de retail y las variables estratégicas de un formato offline y, en tercer lugar, se abordan los puntos centrales de la transformación digital.

Finalmente, se hace hincapié en las influencias de la era digital en el marketing minorista tradicional, repensando y ajustando las variables tradicionales. A partir de ello, entendemos que se requiere repensar la estrategia actual, adaptándose a un entorno cambiante.

Palabras claves: retail; marketing; digital; planificación; estrategia; canal. 


\title{
The transformation of retail marketing into digital era
}

\begin{abstract}
The incorporations of the new information and communication technologies have transformed the way we live, inform ourselves, communicate, buy, how we interact with others, etc. This change has transformed the functional areas of the organization, the marketing area being one of them.

How has retail marketing been transformed in the digital age? The article addresses the concept, the evolution of marketing in an organization and its main variables, secondly the concept of retail and the strategic variables of an offline format, thirdly, it addresses the central points of digital transformation.

Finally, emphasis is placed on the influences of the digital age in traditional retail marketing, rethought and adjusting the traditional variables and like this, it requires rethinking the current strategy, adapting to a changing environment
\end{abstract}

Keywords: retail; marketing; digital; planning; strategy; channel

\section{A transformação do marketing de varejo para a era digital}

Resumo: As incorporações das novas tecnologias de informação e comunicação transformaram a maneira como vivemos, nos informamos, nos comunicamos, compramos, como interagimos com os outros, etc. Essa mudança transformou as áreas funcionais da organização, sendo a área de marketing uma delas.

Como o marketing de varejo foi transformado na era digital? O artigo aborda o conceito, a evolução do marketing em uma organização e suas principais variáveis, em segundo lugar o conceito de varejo e as variáveis estratégicas de um formato offline, em terceiro lugar, aborda os pontos centrais da transformação digital.

Por fim, destaca-se as influências da era digital no marketing de varejo tradicional, repensando e ajustando as variáveis tradicionais e, assim, é necessário repensar a estratégia atual, adaptando-se a um ambiente em mudança.

Palavras-chave: varejo; marketing; digital; planejamento; estratégia; canal.

\section{Introducción}

Uno de los sectores que tuvo mayor transformación en la era digital es el comercio minorista. Los minoristas son negocios que venden sus productos a los consumidores finales u organizaciones para su uso o consumo. En algunos casos, son fabricantes que se integran en forma vertical hacia delante para comercializar sus propios productos y, en otros casos, compran grandes cantidades de mercadería (en volumen) que luego son fraccionadas en pequeñas cantidades y vendidas.

El marketing es una disciplina que centra su atención en el cliente buscando productos para poder satisfacer sus necesidades. A partir de los cambios en el 
entorno -fruto del desarrollo tecnológico- los clientes tienen mayor información disponible, nuevos canales de comunicación, múltiples canales de acceso a la mercadería; asimismo, productos que antes eran físicos pasaron a ser digitales y esto se tradujo en un cambio en el comportamiento de los consumidores.

El objetivo de este artículo, en primer lugar, es realizar un repaso del marketing como forma de gestionar un negocio para la toma de decisiones minoristas que se sustenta en sus pilares estratégicos y su vinculación con la planificación empresarial. En segundo término, analizar algunas innovaciones de la era digital que impactan sobre el retail. En tercer lugar, destacar los principales impactos que han transformado el modelo minorista tradicional en uno dominado por las nuevas tecnologías y, finalmente, enunciar las etapas para diseñar una estrategia digital minorista.

\section{El marketing como filosofía de gestión de un retail}

El marketing como disciplina empresarial no nació hasta la década del 50 (Ryan y Jones, 2009). Sin embargo, las acciones que hoy conocemos como de marketing masivo comenzaron a principios del siglo XX (Zahay, 2015), mientras que la publicidad masiva se desarrolló con la llegada la televisión en la década del 50.

La transformación del sistema productivo y de la economía vino asociado con nuevas formas de comercialización de los productos (Singh Bhatia, 2019; Kotler y Armstrong, 2013; Kerin et al., 2014; Stanton et al., 2007). El enfoque de las empresas estuvo inicialmente en la producción, donde los bienes se fabricaban en masa y se priorizaban los clientes, el bajo precio y su disponibilidad. Luego, el foco de atención se puso en el producto, donde las empresas pusieron más énfasis en la calidad, el rendimiento y las características innovadoras. Posteriormente el eje se centró en la venta. Fruto del aumento de la competencia y la crisis financiera de la década del 20, las empresas se vieron obligadas a enfatizar en la venta y en el desarrollo de promociones agresivas. El problema ya no era cómo producir, sino cómo vender. A continuación, viene la era del marketing, que surge como una práctica cuando los clientes se volvieron más exigentes (luego de la Segunda Guerra Mundial) y las empresas comprendieron que debían centrarse en responder a sus necesidades. Más adelante, en la década del 90, con el surgimiento de la tecnología y los medios electrónicos se da paso a la era de las relaciones, donde los clientes comienzan a ser valorados para construir un vínculo a largo plazo. Finalmente, encontramos la era digital, donde la atención se centra en el marketing en tiempo real basado en un intercambio social en el que la comunicación y las interacciones sociales juegan un papel preponderante (Singh Bhatia, 2019).

El concepto de marketing ha evolucionado con el paso de los años (Ferrell y Hartline, 2018). Algunas definiciones centran su atención en las 4 P tradicionales: product (producto), price (precio), place (plaza), promotion (promoción). Stanton, Etzel y Walker (2007) lo consideran como un sistema de ac- 
tividades ideado para planear bienes o servicios que satisfagan necesidades, asignarles precio, promoverlos y distribuirlos a los mercados meta a fin de lograr los objetivos de la organización. En cambio, para Kotler y Armstrong (2013) es una función que crea valor para el cliente. De esta manera, el marketing es un proceso social y directivo a partir del cual los individuos y las organizaciones obtienen lo que necesitan y desean a través de la creación y el intercambio de valor superior a la competencia. Todo ello, a partir de las relaciones rentables que se generan con los demás. El valor es la relación entre los beneficios y los sacrificios necesarios para obtenerlos. Otros autores como Lamb, Hair y McDaniel (2011) ven al marketing como una filosofía, una actitud, una perspectiva o una orientación gerencial que hace hincapié en la satisfacción del cliente. De esta manera desarrollan actividades y procesos para poner en práctica esta filosofía. Recientemente, la Asociación Americana de Marketing (2020) hizo énfasis en la vinculación entre la oferta de la empresa y la generación de valor para el cliente. Para esta institución, "el marketing es la actividad, el conjunto de instituciones y los procesos para crear, comunicar, entregar e intercambiar ofertas que tienen valor para los clientes, los socios y la sociedad en general". De esta manera, se saca del foco de generación de valor solamente al producto e incluye a todas las actividades, instituciones y procesos que se desarrollan dentro de la organización.

Los objetivos del marketing son reconocer, atraer, actuar, adquirir e involucrar o comprometer a los clientes (Siggelkow y Terwiesch, 2019; Zahay, 2015). Para esto es necesario forjar relaciones, ya que permite transformar las experiencias en relaciones valiosas y duraderas, donde se pone en práctica un intercambio mutuo y en el que el cliente es un socio y participante en los esfuerzos de marketing de la empresa (Zahay, 2015). El proceso de marketing consiste en realizar investigaciones, desarrollar y generar conciencia de marca, generar clientes potenciales, adquirir clientes, comunicar a los clientes, ofrecer productos de mayor valor (up selling), generar ventas cruzadas (cross selling), retener y, finalmente, identificar a los clientes que pueden ser defensores de la marca.

El marketing no se trata de tecnología, sino de personas. La tecnología solo es interesante -desde una perspectiva de marketing- cuando conecta a las personas con otras de manera más eficaz (Ryan y Jones, 2009). El conocimiento del historial pasado de los clientes y sus preferencias permiten la personalización y customización. La personalización significa que usamos información sobre el cliente en nuestras comunicaciones para la venta de productos complementarios. En cambio, customización significa que un producto se crea realmente para ese cliente en función de sus preferencias (Zahay, 2015). La empresa Oxiacycles $^{(1)}$ fabrica bicicletas hechas a medida del cliente. La tecnología hizo que las comunicaciones digitales fueran mejores y se crearon nuevos métodos de comunicación con el cliente. El correo electrónico fue el primer canal digital que surgió en la década del 90 , desde entonces proliferaron nuevos canales 
de comunicación digital que incluyen redes sociales, mensajería instantánea, feeds RSS, etc. (Zahay, 2015). El marketing se trata de lidiar con un mercado en constante cambio, y para comprender el marketing de vanguardia debemos comprender cómo ha evolucionado el mercado en los últimos años (Kotler, et al., 2017).

La dirección estratégica (David, 2003) consiste en el proceso de seleccionar, implantar y evaluar la estrategia de la organización. Para esto se realiza un ajuste estratégico entre las metas de la organización, sus capacidades y sus cambiantes oportunidades de mercado (Kotler y Armstrong, 2013). La dirección estratégica prepara el escenario para el resto de la planeación. El marketing no es un área que se encuentre separada del resto. Las decisiones de marketing deben alinearse con las estrategias institucionales. En ese marco, la planificación de marketing es simplemente una secuencia lógica (Chaffe y Ellis-Chadwick, 2016) y una serie de actividades que conducen al establecimiento de objetivos de marketing y a la formulación de planes para lograrlo.

El marketing actúa sobre los bienes, servicios, ciudades e ideas al emplear estrategias de comunicación, distribución y precio (Lamb et al., 2011). El comercio minorista es una de las principales actividades económicas que tiene un país y desempeña una función imprescindible en todas las sociedades evolucionadas al encargarse de la venta de bienes y servicios directamente al consumidor final.

El comercio minorista tiene muchas formas de denominación, entre ellas retail, tienda al por menor, minoristas o detallistas. Se cree que el término "minorista" deriva de la antigua palabra francesa "retailler" que significa "un pedazo de" o "cortar". Los minoristas compran grandes cantidades de productos que luego son fraccionados en pequeñas cantidades para ser vendidos a los clientes. Lusch, citado por Goworek y McGoldrick (2015), señala que la venta minorista consiste en las actividades finales y los pasos necesarios, ya sea para colocar un producto en manos del consumidor o para brindarle un servicio. El negocio minorista es aquella organización que realiza las actividades de compra de los bienes o insumos, ya sea a un fabricante, mayorista o distribuidor, y los vende al consumidor final. El comercio minorista -al encontrarse entre la producción y el consumo- cumple un rol preponderante en la economía. Desde el punto de vista del marketing minorista se establece el producto correcto, en el lugar adecuado, en el momento oportuno, en la cantidad suficiente y a un precio justo.

El proceso de planificación estratégica minorista es el conjunto de pasos que realiza un minorista para formular, implementar y evaluar una estrategia. Describe cómo los minoristas seleccionan los segmentos de mercado objetivo, determinan el formato minorista apropiado y crean ventajas competitivas sostenibles. Una estrategia minorista es el plan general o marco de acción que guía a una empresa (Berman y Evans, 2013), que describe la misión, los objetivos, el mercado, las actividades generales y específicas y los mecanismos de control. En la misma sintonía, Levy, Weitz y Grewal (2014) señalan que es una declaración que 
identifica el mercado objetivo, el formato y los recursos que el minorista planea utilizar para satisfacer las necesidades del mercado objetivo y las bases sobre las cuales el minorista planea construir una ventaja competitiva a través de la generación de valor.

El proceso inicia con el análisis de la situación, que es una evaluación sincera de las oportunidades, amenazas, debilidades y fortalezas que tiene y enfrenta un minorista. Una herramienta comúnmente utilizada es la matriz DAFO, que busca responder a dos preguntas generales: ¿cuál es el estado actual de la empresa? y ¿̇en qué dirección debería dirigirse? Una buena estrategia se anticipa y se adapta tanto a las oportunidades como a las amenazas en un entorno dinámico. Las oportunidades son aperturas de mercado que existen porque otros minoristas aún no las han capitalizado. Las amenazas son factores ambientales y del mercado que pueden afectar negativamente a los minoristas si no reaccionan a ellos. A partir de esto, deben identificarse oportunidades que permitan sostener, ampliar o lograr una posición competitiva del retail. Después del análisis de situación, el minorista debe establecer los objetivos, que son metas de desempeño a mediano y corto plazo que espera alcanzar. Todo ello ayuda a moldear una estrategia y traduce la misión organizacional en acción. Una empresa puede perseguir objetivos relacionados con una o más de estas áreas: ventas, margen de contribución, ganancias, satisfacción del cliente y posicionamiento de marca (Berman y Evans, 2013).

Una etapa clave del proceso es la selección del grupo de clientes que va a atender y que se llama mercado objetivo. Al seleccionar su mercado objetivo, un retail puede utilizar una de tres estrategias de cobertura: mercado indiferenciado con la venta de bienes y servicios a un amplio espectro de clientes; mercado concentrado que se enfoca en un grupo específico; y mercado diferenciado, dirigido a dos o más segmentos de clientes distintos, con distinto mix de venta minorista para cada grupo.

Luego de la selección del mercado meta, el minorista debe definir su propuesta de valor a partir de dos componentes: las variables propias del negocio que la afectan directamente y aquellos a los que el minorista debe adaptarse. Las primeras se denominan variables controlables y las segundas se denominan variables incontrolables (Berman y Evans, 2013). Las decisiones sobre las variables controlables de una estrategia minorista consisten en la ubicación, el surtido, la administración del negocio, el formato de atención, la política de precios, la publicidad y promoción, el merchandising y el servicio al cliente. Como consecuencia de estas decisiones, el minorista define el formato de negocios a partir de la naturaleza de la combinación minorista que utilizará para satisfacer las necesidades del mercado objetivo (tiendas de descuento, supermercados, tiendas de conveniencia, de departamento, autoservicio, especializada, entre otras). Por su parte, las variables incontrolables de una estrategia consisten en el cliente, la competencia, la tecnología, las condiciones económicas, la estacionalidad y las restricciones legales. Los minoristas deben ajustar la propuesta de 
valor a las variables incontrolables que se presentan. Si bien la estrategia debe darle previsibilidad a la organización, algunos autores como Mintzberg, Queen y Voyer (1997) y Chaffe y Ellis-Chadwick (2016) resaltan la importancia de la estrategia emergente en un entorno cambiante y dinámico con gran influencia de la tecnología.

Finalmente, en la fase de control se lleva a cabo una revisión de las estrategias y las tácticas que se evalúan en relación con los objetivos y el mercado objetivo. Este procedimiento se denomina "auditoría minorista" y es un proceso sistemático para analizar el desempeño. En cada etapa, la gerencia recibe señales o pistas, conocidas como retroalimentación, sobre el éxito o el fracaso de la estrategia. Pero si el minorista no logra sus objetivos se requiere un nuevo análisis. Por lo general, este nuevo análisis comienza con la revisión de los programas de implementación, pero puede indicar que la estrategia (o incluso la declaración de misión) debe reconsiderarse. Esta conclusión da como resultado el inicio de un nuevo proceso de planificación que incluye un nuevo análisis DAFO (Levy et al., 2014).

\section{La era digital y el marketing minorista}

El surgimiento y uso masivo de la tecnología digital ha cambiado la manera en la que vive la población. Con esto, nos referimos a cómo se comunica, cómo se obtienen datos y se los transforma en información, cómo se aprende y cómo se accede a nuevos bienes y servicios (Kotler y Armstrong, 2013). Cuando emerge una nueva tecnología, inicialmente es dominio exclusivo de los tecnólogos, luego la tecnología gana una posición más firme en el mercado y comienza a ser más popular poniéndola en el radar de otras disciplinas como el marketing. Tal como señala McGovern, citado por Charlesworth (2018), lo digital es el agente de transformación, no la transformación en sí misma. La conectividad es -posiblemente- el factor de cambio más importante en la historia del marketing (Kotler et al., 2017). La conectividad reduce significativamente los costos de interacción entre los distintos actores relacionados en el proceso comercial: los minoristas y sus empleados, los proveedores, los clientes y otros stakeholders. Asimismo, reduce las barreras para ingresar a nuevos mercados, permite el desarrollo simultáneo de productos y acorta el período de tiempo para el posicionamiento de la marca.

Los desarrollos en tecnología y la evolución del retail marketing están indisolublemente entrelazados (Ryan y Jones, 2009). Varias tecnologías juntas facilitan el desarrollo del marketing digital minorista: internet, la informática generalizada, la tecnología de bases de datos y el big data (Zahay, 2015; Charlesworth, 2018). Internet permitió que la tecnología estuviera ampliamente disponible para la mayoría de la población y su mayor impacto se puede atribuir al auge de las tecnologías de búsqueda (Singh Bhatia, 2019). El objetivo principal de un motor de búsqueda es organizar internet y proporcionar una estructura coherente para acceder de manera fácil a la información a partir de palabras claves 
específicas, por ejemplo, viaje a Mar Del Plata. Con ello proliferaron los medios de comunicación y otras herramientas para compartir videos, redes sociales y aplicaciones para teléfonos móviles. El retail marketing cada vez depende más del uso de la tecnología (Brinker y McLellan, 2014). Las herramientas y estrategias que eran de vanguardia hace solo unos años se están volviendo obsoletas rápidamente (De Swaan Arons et al., 2014; Edelman, 2010) y harán obsoletas las que usamos ahora en muy poco tiempo.

Como consecuencia de esta transformación digital, los especialistas en marketing minorista están repletos de datos de clientes que son utilizados para aprender y comprender el comportamiento de compra y consumo a fin de diseñar estrategias comerciales efectivas (De Swaan Arons et al., 2014; Kotler y Armstrong, 2013). En este contexto, las nuevas tecnologías pueden abrir nuevas oportunidades de mercado para los minoristas. El conocimiento de los clientes permite seleccionar los productos más comprados, orientar los mensajes, diseñar el servicio según las preferencias de los clientes, construir relaciones a largo plazo y mejorar el nivel de servicio posventa, entre otras. Sin embargo, a partir del cambio digital, de las nuevas plataformas y de las redes sociales, las empresas no pueden controlar las conversaciones de los clientes sobre las marcas. Los consumidores toman el contenido generado por una empresa, lo llevan con ellos a donde vayan y lo comparten con otras personas.

\section{La influencia del marketing digital en el marketing minorista}

El marketing digital se puede definir como el uso de cualquier tecnología digital (medios electrónicos y plataformas) para facilitar el proceso de marketing con el objetivo de promover la interacción y el compromiso del cliente y lograr los objetivos deseados para una organización (Zahay, 2015; Chaffe y Ellis-Chadwick, 2016; Chaffey y Smith, 2017). Singh Bhatia (2019) cita el concepto de marketing digital del Institute of Direct and Digital Marketing (IDM), que incluye la gestión y ejecución de marketing, al utilizar medios electrónicos como la web, el correo electrónico, la TV interactiva, los medios inalámbricos, junto con datos digitales sobre las características y el comportamiento de los clientes. Algunos confunden al marketing digital con el comercio electrónico, sin embargo, el marketing digital es más amplio que el comercio electrónico, ya que no se limita a las transacciones entre una organización y sus partes interesadas, sino que incluye todos los procesos relacionados con el marketing (Chaffey y Smith, 2017). Entre sus objetivos podemos destacar: acercarse a los clientes y comprenderlos mejor, agregar valor a los productos, ampliar los canales de distribución e impulsar las ventas mediante la ejecución de campañas que utilizan canales digitales (Chaffey y Smith, 2017).

Para Levy, Weitz y Grewal (2014) son cuatro las variables útiles para evaluar la propuesta de valor de un minorista offline: el surtido ofrecido, la amplitud y variedad de los bienes, el nivel de servicio al cliente y el precio. Por su parte, Gilbert (2003) resalta las comodidades que intenta brindar un minorista en 
términos de ubicación (localización y accesibilidad), pago, crédito, instalaciones, gama de productos y servicio posventa. Asimismo, Berman y Evans (2013) sostienen que un formato minorista surge de las decisiones que toman los responsables mediante la combinación particular de la ubicación, procedimientos operativos, bienes y servicios ofrecidos, precio, ambiente de la tienda, servicio al cliente y políticas de promociones.

A partir de la era digital, muchas de estas variables que eran determinantes dejaron de tener un peso específico en la decisión del cliente para la elección de un formato minorista. Tal como señalan Kotler y Stigliano (2020), la revolución digital ha modificado muchos de los supuestos en los que se ha basado el comercio minorista tradicional en las últimas décadas.

La revolución digital generó grandes transformaciones en los pilares estratégicos tradicionales de un retail. Sin embargo, en palabras de Kotler y Stigliano (2020), es exagerado hablar de un apocalipsis. Añaden que los modelos tradicionales deben revisarse a partir de los cambios introducidos por la era digital. La siguiente figura es una lista de las principales influencias del marketing digital en un retail.

Figura 1: Influencias del marketing digital en el retail

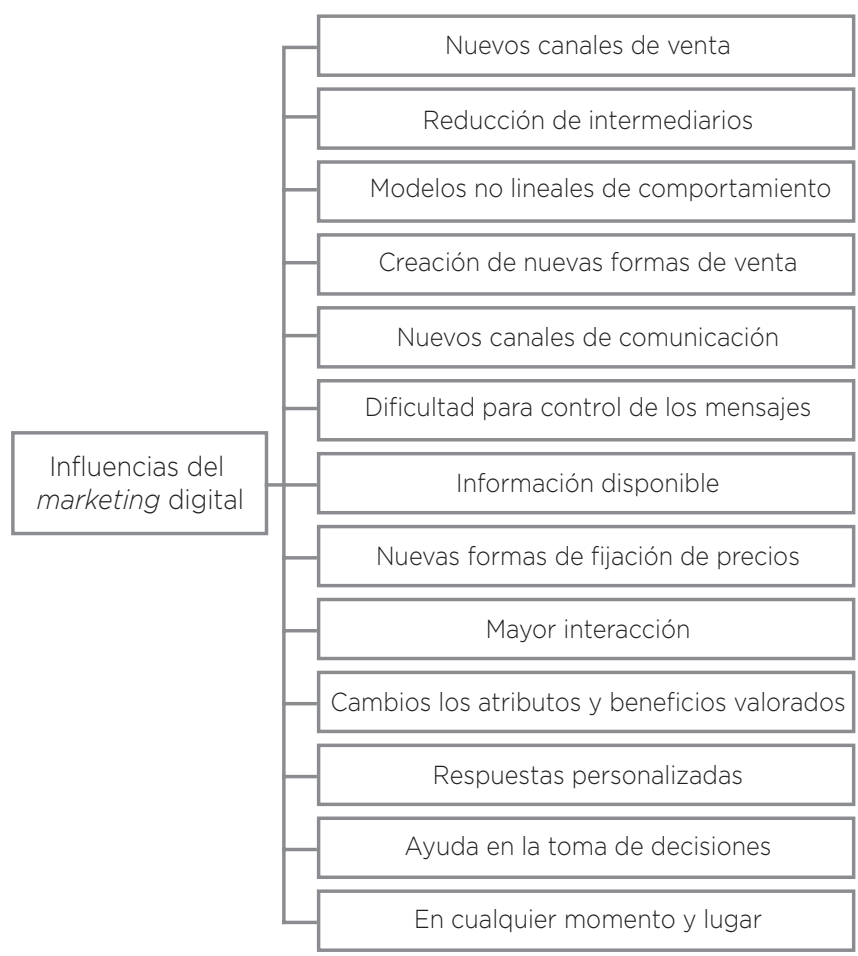

Fuente: Elaboración propia. 
Uno de los principales componentes de la mezcla de marketing que se vio afectado por la era digital fue la distribución física y comercial (plaza) a partir de la creación de nuevos canales de venta. Un producto puede seguir diversas rutas para llegar a su consumidor final. Los productos de la empresa Lacoste pueden encontrarse en los locales offline que llevan su mismo nombre y en minoristas que venden productos multimarca; además, se puede visitar el sitio web $^{(2)}$ para hacer compras online y en otras plataformas digitales como Dafiti(3) y Mercado Libre, ${ }^{(4)}$ entre otras. El supermercado Vea, además de sus puntos de venta, posee un sitio(5) para hacer las compras. Las empresas deben buscar el canal más eficiente entre todas las alternativas disponibles (Lamb et al., 2011). Por ejemplo, la marca Infinit de lentes de sol y receta tiene su sitio web(6) que permite comprar a los clientes los productos. Sin embargo, utiliza distribuidores para la venta a las ópticas que luego vende los productos al consumidor final también. En el mundo existen millones de personas que se encuentran conectadas a través de una computadora, un dispositivo móvil o cualquier otra forma de conectarse que pueda surgir. Las buenas estrategias de distribución pueden contribuir fuertemente para brindar mayor valor al cliente y crean una ventaja competitiva para la empresa (Kotler y Armstrong, 2013). La tecnología electrónica interactiva ha hecho posible desarrollar nuevos canales para hacer que los bienes y servicios estén disponibles para el consumo o para el uso de los consumidores o compradores organizacionales. Un pasaje en avión puede comprarse a través de una agencia de viajes, en el sitio de la propia aerolínea(7) y en plataformas como Despegar.(8) A partir de la tecnología digital, los minoristas pueden llegar a clientes potenciales a través de un conjunto de plataformas y dispositivos electrónicos interactivos, intuitivos y avanzados, que van más allá de la web y el correo electrónico, como los teléfonos móviles, PDA, TV interactiva, aplicaciones (Dafiti, Mercado Libre, Despegar, Amazon, Wish) y redes sociales. ${ }^{(9)}$ Si bien la tecnología es fundamental para su desarrollo, según Ryan y Jones (2009) el marketing digital minorista no se trata de tecnología, sino que se trata de personas. En ese sentido, es similar al marketing tradicional, es decir, se trata de personas (a través de empresas) que se conectan con otras personas para construir relaciones y, en última instancia, impulsar las ventas.

Una característica única de estos canales es que combinan intermediarios electrónicos y convencionales para crear utilidad en tiempo, lugar, forma y pose-

(2) Veáse https://www.lacoste.com/ar

(3) Veáse https://www.dafiti.com.ar/

(4) Veáse https://ropa.mercadolibre.com.ar

(5) Veáse https://www.veadigital.com.ar/

(6) Veáse https://www.infinit.la/

(7) Veáse https://www.aerolineas.com.ar/

(8) Veáse https://www.despegar.com.ar

(9) Veáse las cuentas de Instagram de: @dafiti_arg, @aerolineas_argentinas y @despegar

168 | Ediciones SAIJ < Ministerio de Justicia y Derechos Humanos de la Nación 
sión para los compradores (Kerin et al., 2014). Si bien el marketing online se ha convertido en un modelo de negocio exitoso para algunas empresas, para otras seguirá siendo solo un canal o medio más, en una mezcla de marketing totalmente integrada. Para Kotler y Stigliano (2020), en algunos casos, los locales desempeñarán una función de sala de exposición para transmitir un experiencia, promocionar y añadir espectacularidad a un producto. La empresa Sony, además de tener su punto de venta con productos exclusivos, Ilamada Sony Store, y vender a través de minoristas de electrónica, tiene su tienda virtual.(10) En otros casos, empresas que surgieron totalmente en línea como Amazon y Alibaba abrieron puntos de venta físicos en varias ciudades de Estados Unidos. Para Kotler, Kartajaya y Setiawan (2017), los canales van a coexistir para ofrecer la mejor experiencia al cliente y así lograr una combinación perfecta de experiencias en línea y fuera de línea. Además, el mundo offline puede ser una fuerte herramienta de diferenciación en un mundo cada vez más online. La empresa deberá decidir si internet será solo otro canal de ventas o si cambiará fundamentalmente la forma en la que vende a sus clientes. Al respecto, Chaffe y Ellis-Chadwick (2016) plantean algunos interrogantes que tienen que hacerse las empresas, a saber: ¿internet complementará principalmente a los otros canales de la empresa o los reemplazará?

Las ventas al detalle en línea, o e-tailing, son un tipo de ventas disponibles para los consumidores que tienen acceso a internet (Lamb et al., 2011). El Louvre, las Pirámides y muchas más atracciones (recitales, obras de teatro) ahora pueden extender su distribución tanto del punto de compra -es decir, comprar una entrada (https://www.ticketek.com.ar/, https://www.plateanet.com, https://www.atrapalo.com.ar) para obras de teatro o música-, como del punto de consumo, permitiendo disfrutar de la vista desde la propia casa -por ejemplo, el museo Louvre (https://www.louvre.fr) o la Capilla Sixtina (http://www.museivaticani.va) (Chaffey y Smith, 2017). Cuando un productor elige dos o más canales para entregar el mismo producto a los mercados meta, a este arreglo se le llama "distribución dual o distribución múltiple": Empresas como Lacoste, Daniel Hechter, Disco, Vea, Prune, entre otros, poseen este sistema. Conforme más personas tienen acceso a internet y adoptan las compras en línea, un número cada vez mayor de minoristas utilizan canales múltiples de distribución (Lamb et al., 2011). Los clientes ahora pueden comprar boletos de avión, planear un crucero de vacaciones, reservar una habitación de hotel, pagar las cuentas y recibir periódicos electrónicos. Otra práctica surgida de la era digital es la omnicanalidad como una gran oportunidad de crecimiento. En esta modalidad se integran múltiples canales para crear una experiencia uniforme al cliente, y requiere que las organizaciones rompan los silos de canales y unifiquen sus objetivos y estrategias.

En los primeros 50 años de la disciplina, las herramientas de marketing se desarrollaron en la industria en el contexto del marketing masivo: dirigidas a

(10) Veáse https://store.sony.com.ar/ 
mercados amplios con mensajes y ofertas estandarizados y distribuidos a través de intermediarios (distribuidores, mayoristas, minoristas). Como consecuencia del desarrollo de nuevos canales de distribución, muchas empresas están adoptando el uso de canales directos que permiten reducir intermediarios (Kotler y Armstrong, 2013). De esta manera se pueden comprar productos directamente al fabricante o prestador el servicio: usamos Tienda León ${ }^{(11)}$ para un transporte para ir a Ezeiza, Arcor en casa ${ }^{(12)}$ para comprar productos alimenticios y la Sony Store(13) para comprar algún producto electrónico. Los productores utilizan el canal directo para vender sin intermediarios a los consumidores. Las actividades de telemarketing, pedidos por correo electrónico, compras por catálogo y formas de ventas minoristas electrónicas como portales web y redes sociales (Lamb et al., 2011; Singh Bhatia, 2019; Zahay, 2015) son un buen ejemplo de este tipo de estructura de canal.

El formato de compra tradicional suponía el uso de un modelo racional en el que la organización debía generar primero la conciencia o atención, luego la familiaridad, en tercer lugar la consideración y, finalmente, la compra. Con la irrupción de los diferentes puntos de contacto, el modelo lineal en el desarrollo de la estrategia comienza a desaparecer (Kotler y Stigliano, 2020).

En la economía colaborativa, el concepto de distribución más potente es la distribución peer-to-peer. Esta es una forma de venta entre pares. Organizaciones como E-bay, Alibaba, Airbnb, Uber, Zipcar y Lending Club, Pedidos Ya, Rappi, Mercado Libre y Amazon están interrumpiendo las industrias de la venta minorista, hoteles, taxis, alquiler de automóviles y banca, respectivamente. Proporcionan a los clientes un fácil acceso a los bienes y servicios que no son de su propiedad, sino de otros clientes, donde las organizaciones actúan como intermediarios.

La comunicación es el proceso por el cual intercambiamos o compartimos significados mediante un conjunto común de símbolos (Lamb et al., 2011). Sirve para informar, persuadir y recordar a los clientes potenciales acerca de un producto con el fin de influir en su actitud hacia la marca y se debería diferenciar de las herramientas como la publicidad, la promoción, las relaciones públicas (Kerin et al., 2014) y los medios de comunicación. La incorporación de nuevos canales de comunicación, gracias a la tecnología digital, sirve para desarrollar una conexión duradera con los clientes en forma más sencilla y eficiente. A través de múltiples canales, en línea y fuera de línea, los clientes continúan expuestos a todo: características del producto, promesas de marca y acciones de ventas. Los nuevos canales de comunicación como el correo electrónico, sitios web de marca, aplicaciones móviles, redes sociales, blogs y pódcast -fruto del avance en la tecnología de las comunicaciones- provocan cambios sustantivos en las

(11) Véase https://www.tiendaleon.com

(12) Véase https://arcorencasa.com/

(13) Véase https://store.sony.com.ar/ 
empresas y en los clientes (Kotler y Armstrong, 2013). En algunas ocasiones, confundidos por mensajes publicitarios demasiado buenos para ser verdaderos, los clientes a menudo los ignoran y, en cambio, recurren a fuentes confiables de consejos: su círculo social de amigos y familiares (Kotler et al., 2017).

Con relación a los medios, el sitio web es el centro de su mundo digital y quizás el elemento más importante de toda su estrategia de marketing digital. La optimización de motores de búsqueda (SEO) forma parte del proceso de alinear el contenido del sitio web con lo que sus consumidores buscan, de forma tal de hacerlo más accesible para el cliente. La publicidad paga por clic (PPC) ofrece una forma de acceder a las páginas de búsqueda a través de palabras clave o frases elegidas. Esta es una forma extremadamente eficaz de generar tráfico en los motores de búsqueda de manera rápida. El marketing de afiliados y asociaciones estratégicas contempla realizar un acuerdo con otras organizaciones y sitios web en relaciones para promover sus bienes o servicios. El correo electrónico, el abuelo del marketing en internet (Ryan y Jones, 2009), está en crisis a raíz del bombardeo de spam, pero sigue siendo una herramienta importante del marketing digital, particularmente cuando se trata de mantener relaciones continuas con clientes actuales y potenciales que optaron por recibir información. Los medios sociales, a los que en ocasiones se les llama medios de contenido generado por el usuario o por el consumidor, incluyen blogs, pódcasts, foros, recorridos de video interactivo, redes sociales y mundos virtuales (Lamb et al., 2011). Dentro de las redes más conocidas podemos resaltar a Instagram, Facebook, Twitter, LinkedIn, YouTube y Snapchat. Las redes sociales pueden ofrecer publicidad altamente dirigida a grupos especializados en función de la información de perfil que ofrecen voluntariamente. Una nueva manera de llegar al cliente crece así a pasos agigantados. El marketing de contenido es un enfoque que implica crear, seleccionar, distribuir y ampliar contenido que sea interesante, relevante y útil para un grupo de audiencia claramente definido con el fin de crear conversaciones sobre este. El marketing de contenidos también se considera otra forma de periodismo de marca y publicación de marca que crea conexiones más profundas entre marcas y clientes (Kotler et al., 2017).

En los medios digitales es a menudo el cliente quien inicia el contacto con una marca y quien busca información o una experiencia al visitar un sitio web de la empresa. En otras palabras, es un mecanismo de atracción en el que es particularmente importante tener una buena visibilidad en los motores de búsqueda cuando los clientes ingresan términos relevantes para los productos de una empresa. Este nuevo y poderoso enfoque se conoce comúnmente como inbound marketing o marketing entrante. El marketing de contenido y de búsqueda se puede utilizar para dirigirse a clientes potenciales con una necesidad definida: son proactivos y se seleccionan por sí mismos (Chaffey y Smith, 2017).

La planeación de medios es una serie de decisiones que las empresas toman en relación con la selección y empleo de los canales que permite comunicar de forma óptima y eficaz sobre los costos, el mensaje y al mercado meta. 
Las decisiones de la mezcla de medios se basan en varios factores: costo por contacto, alcance, frecuencia, consideraciones del público meta, flexibilidad del medio, nivel de ruido y lapso de vida del medio (Lamb et al., 2011). En primera instancia, las empresas offline comenzaron a utilizar canales digitales (llamados "no tradicionales") para lograr una mayor cobertura en su comunicación; sin embargo, las empresas que desarrollan todas sus actividades en plataformas digitales como Netflix, Amazon Prime, Disney y Pedidos Ya hacen publicidad en medios tradicionales, como por ejemplo la televisión, para difundir sus productos.

En el marketing tradicional, las empresas buscan controlar el contenido del mensaje que ve la audiencia (Barker et al., 2013). En la forma tradicional de marketing, la información que se entregaba a los clientes era decidida, seleccionada o creada por un grupo de expertos en medios (Singh Bhatia, 2019). Las empresas que buscaban transmitir un mensaje a una gran audiencia solo tenían una opción real. La misma era cubrir a una gran franja de clientes de manera simultánea al utilizar la comunicación masiva unidireccional, entre ellas la televisión, la radio, los diarios y los folletos (Rust et al., 2010). En los medios tradicionales predominan los medios push en los que el mensaje se transmite de la empresa al cliente. Durante este proceso, hay una interacción limitada con el cliente, aunque se fomenta la interacción, en algunos casos, como el anuncio de respuesta directa o la campaña de pedido por correo. En cambio, en las redes sociales (Instagram, Facebook, Twitter, Linkedln, YouTube y Snapchat) se enfatiza en la contribución de la audiencia, donde la empresa pierde el control del contenido por lo que, para construir su imagen, las empresas deben desarrollar relaciones de confianza con su audiencia meta. En estas redes o plataformas, los clientes realizan comentarios, le dan valoración a las marcas o productos (estrellas, me gusta). El anonimato es otro rasgo que puede tener un efecto profundo en el comportamiento del cliente (Ryan y Jones, 2009). En línea son libres de hacer y decir lo que quieran, con escasa consideración por el decoro social que impera en la vida real. En una tienda física, los clientes esperarán el servicio y, a menudo, soportarán una experiencia negativa para obtener lo que desean. En línea la paciencia es menor. Con tecnologías disruptivas, ciclos de productos más cortos y tendencias que cambian rápidamente, una marca debe ser lo suficientemente dinámica como para comportarse de determinadas formas según la situación lo requiera. Sin embargo, lo que debe permanecer constante son los caracteres y códigos de la marca (Kotler et al., 2017).

En internet, la información está disponible. Esto no significa -necesariamenteque la empresa tiene que tener el producto más barato, pero para competir debe asegurarse de que su propuesta de valor general para el cliente sea atractiva en relación con el precio. Existen aplicaciones como Comprando, en la que un cliente, al escanear el código de barra de un producto, puede saber el precio del mismo en diferentes minoristas y le indica la localización más cercana del mismo. La democratización, por la reducción de los costos y simplificación del 
uso de la tecnología, permite que el alcance de los beneficios de la era digital y el acceso a la información sea cada vez mayor (Kotler y Stigliano, 2020). Incluso, en muchas plataformas, los clientes pueden realizar comparación de precios (despegar.com, Tripadvisor). Al tener la posibilidad de realizar comparaciones de precios, los clientes tienen una idea clara de la cotización del competidor y el producto de la empresa.

El precio y la nueva forma de fijación han variado con respecto al enfoque tradicional por la reducción de intermediarios a través de canales digitales, por el conocimiento sobre el comportamiento del cliente y por las nuevas formas de interacción. Las dos razones clave por las que la gente compra productos en línea son la conveniencia y el costo (Singh Bhatia, 2019). Por un lado, las industrias adoptan canales directos, ya que ven la oportunidad de reducir los intermediarios y proporcionar el mismo producto a precios atractivos y competitivos a más personas de las que pueden hacerlo actualmente a través de los canales tradicionales. Además, el conocimiento sobre los hábitos y la propensión de compra de los clientes ayuda a crear precios específicos para grupos de personas y para canales. La fijación de precios dinámicos establece precios flexibles en función de la demanda del mercado y la utilización de la capacidad. Los minoristas en línea, por ejemplo, recopilan una gran cantidad de datos, lo que les permite realizar análisis de big data y, a su vez, ofrecer un precio único para cada cliente. Con precios dinámicos, las empresas pueden optimizar la rentabilidad al cobrar a diferentes clientes de manera distinta según los patrones de compra históricos, la proximidad de las ubicaciones de las tiendas y otros aspectos del perfil para, de esa manera, generar mayor valor agregado para satisfacer las necesidades individualizadas. Otro aspecto relevante es la capacidad para que los consumidores establezcan y negocien precios en línea, por ejemplo, en sitios de subasta y también en plataformas de compra y venta, donde los clientes pueden intercambiar productos a precios autoestablecidos. En la red también prolifera el mecanismo freemium para aquellos consumidores que desean probar el producto de forma gratuita antes de que las empresas puedan empezar a cobrar (descarga de una parte de un libro en Amazon, Google Play Books).

El marketing digital minorista utiliza información de los clientes para rastrear y medir su respuesta (Zahay, 2015). Las bases de datos son cruciales en el desarrollo del concepto de marketing interactivo. Para poder acercarse y comprenderlos mejor, el marketing digital busca mayor interacción con el cliente. La interacción es la manera en la que cualquier cliente potencial llega a conocer y se relaciona con la prestación del servicio que hace el minorista y con los productos que vende, con la intención de obtener información y realizar una transacción comercial. El desarrollo de sistemas Customer Relationship Management (CRM) resulta una herramienta útil para conocer al cliente. Antes se utilizaban medios tradicionales (como televisión, radio, periódicos) que no son interactivos, en cambio, las plataformas digitales permiten el ida y vuelta entre 
minorista y el cliente y con otros usuarios. Por las propias acciones que realizan los clientes en línea, por ejemplo, sitios web visitados, términos de búsqueda utilizados en motores de búsqueda, productos vistos y/o comprados y, a partir de las cookies (son archivos que crean los sitios que visita, guardando información de la navegación), luego de la visita en un sitio al usuario le aparecen publicidades de los productos que miró. A esto se lo denomina retargeting y tiene por objeto recordar a los usuarios interesados los productos que en alguna oportunidad buscó. Otro aspecto a tener en cuenta son las discusiones en redes sociales, donde el cliente indica sus intereses y preferencias de compra (Charlesworth, 2018).

Una de las primeras decisiones en la estrategia de marketing a considerar es la selección del mercado meta. En relación a los consumidores digitales, Ryan y Jones (2009) señalan que no existen. La segmentación y la focalización son decisiones unilaterales que toman las empresas sin el consentimiento de los clientes. Los clientes y clientes potenciales que se encuentran en línea son los mismos que entran en la tienda o llaman por teléfono. Son personas como todos los demás. El acceso a medios electrónicos ha permitido que los clientes se conecten en cualquier momento y desde cualquier lugar mediante sus computadoras, teléfonos inteligentes, tablets o incluso sus televisores (Kotler y Armstrong, 2013). Pueden elegir el contenido que quieran, cuando y de la forma en que lo deseen. Incluso pueden crear los suyos propios y compartirlos con sus amigos, sus compañeros y con el mundo de forma gratuita a través de aplicaciones como YouTube, vivos de Instagram o historias (Ryan y Jones, 2009). Esta forma de conexión ha cambiado el valor que le dan los clientes a los atributos y beneficios brindados por las empresas: comodidad, velocidad, precio, información de un bien o servicio y el servicio posventa. Estas variables son diferentes a las valoradas por los clientes en formatos offline. Como resultado, las empresas tienen una forma nueva para crear valor para los clientes y construir relaciones con ellos (Kotler y Armstrong, 2013). Es fundamental comprender a las personas y su comportamiento de compra y consumo, y aquí es donde la noción del consumidor digital tiene cierto peso, porque el comportamiento del consumidor está cambiando fruto de la tecnología. A los consumidores digitales les gusta manifestarse, no se guardan las deficiencias de su producto o negocio para sí mismos. En plataformas de entretenimiento como Netflix podemos incluir calificaciones, en Despegar se pueden añadir calificaciones numéricas. Los usuarios han pasado de ser un receptor dócil a un buscador activo de información (Singh Bhatia, 2019). El marketing digital no se trata de aprender cómo funciona la tecnología, sino de comprender cómo las personas están utilizando esa tecnología y cómo se la puede aprovechar para interactuar con ellos de manera más efectiva (Ryan y Jones, 2009).

Un buen producto es la piedra fundamental de toda estrategia de marketing exitosa y se justifica a partir de la propuesta de valor único para los clientes. En el caso de un minorista, un servicio que ofrece beneficios y satisface una 
necesidad real en el mercado deja en el cliente una percepción genuina de valor. Las empresas se benefician de dos capacidades que les ofrece la tecnología: la interactividad y la individualidad (Kerin et al., 2014). Los tableros de elección y los filtros colaborativos son esfuerzos para proporcionar respuestas personalizadas a las necesidades de los clientes. En la práctica iniciada por el cliente, se genera contenido hecho a la medida de las necesidades y preferencias del sitio web de una empresa. Una oferta se diferencia de su forma tradicional en el mecanismo en que se desarrolla e integra los aspectos intangibles o de servicio (Singh Bhatia, 2019). Esto puede realizarse como nuevos productos digitales (libros, música, entretenimiento, juegos) o ser una extensión del producto en sí, para facilitar al cliente el acceso a la información, el servicio posventa, la interacción y, en última instancia, la compra del producto principal. La empresa DeWalt, que vende herramientas en su sitio web, ${ }^{(14)}$ tiene un espacio de servicio donde el usuario puede descargar los manuales de funcionamiento y un canal de YouTube llamado Dewalt Latam donde se pueden encontrar videos sobre el funcionamiento de las herramientas. La cocreación es la estrategia de desarrollo de nuevos productos mediante la creación conjunta y la participación de los clientes en la etapa de desarrollo de la idea. De esta manera, las empresas pueden mejorar la tasa de éxito de nuevos productos. La cocreación también permite a los clientes personalizar productos y crear así propuestas de valor que sean superiores (Kotler et al., 2017).

En las tiendas offline, los clientes se enfrentan con la tarea de analizar una gran cantidad de opciones y tomar una decisión de compra. Al ingresar a Pedidos Ya, el cliente puede ver el último pedido realizado y se le da la opción de repetirlo. De esta manera, la empresa puede ayudar a los clientes a descubrir sus preferencias. Kotler, Kartajaya y Setiawan (2017) destacan la importancia de las tecnologías de sensores e identificación por radiofrecuencia que brindan soluciones a este problema. De esta manera, la tecnología permite comunicarse con los teléfonos inteligentes de los clientes mediante la tecnología Bluetooth, creando conexiones directas.

En relación a la información, las empresas de cualquier parte del mundo proporcionan información directa a los clientes de cualquier parte, en cualquier momento. Esto se da porque los horarios de atención al público y las limitaciones geográficas dejaron de existir. Los sitios web y las redes sociales proporcionan información. La utilidad de posesión, el proporcionar un bien o un servicio a los clientes para que lo puedan poseer o usar es acelerada. Un cliente puede comprar un libro digital a través de alguna plataforma y comenzar a leerlo en ese instante. De la misma manera, puede usar un software o una plataforma de streaming para ver una serie o película, realizar la reserva de un hotel o la compra de un boleto de avión.

(14) Véase https://ar.dewalt.global 


\section{Estrategia de marketing digital para un retail}

El desarrollo de la estrategia de marketing digital minorista puede pensarse a través del modelo ASCOR (Singh Bhatia, 2019). El mismo es un acrónimo que representa las cinco fases del proceso: assessment (evaluación), strategy (estrategia), communication y channel (comunicación y canal), operations (operaciones) y refinement (refinamiento).

Figura 2: Fases del proceso de marketing digital en un retail

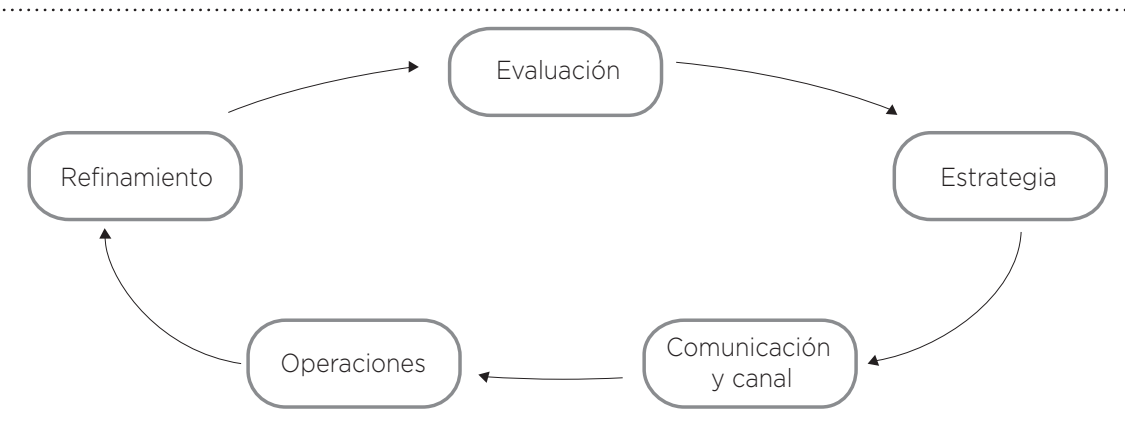

Fuente: Singh Bhatia, 2019

La primera fase implica la evaluación y el análisis del entorno actual del mercado externo, interno y la definición de los objetivos de la empresa. La estrategia digital desarrollada por un minorista dependerá de las influencias externas e internas. Esta etapa contempla la realización de una investigación de mercado necesaria para evaluar las tendencias del comportamiento actual del mercado (preferencias de consumo, nuevas tecnologías utilizadas, mecanismos de relacionamiento). Las actividades incluyen un análisis del entorno macro-micro (entidades que rodean e influyen en la empresa, sus productos, las operaciones y las oportunidades) y el análisis de la situación del mercado (incluye análisis de mercado y competencia, específico de la industria donde la empresa y sus productos están funcionando). En cambio, el análisis interno implica un análisis de los factores propios de una empresa que repercuten en el desarrollo de una estrategia digital y que están bajo su control.

En la segunda fase, la empresa debe establecer los objetivos y formular la estrategia de marketing digital basados en el estado digital específico de la empresa. Los objetivos son los resultados esperados que se pueden agrupar en función de la adquisición, la conversión, la retención y el crecimiento de los clientes. El resultado de la estrategia digital es una serie de iniciativas de marketing digital para la adquisición, conversión, retención y crecimiento de clientes y el desarrollo de propuestas y experiencias (Chaffe y Ellis-Chadwick, 2016). La propuesta de valor del minorista es una declaración de los resultados tangibles e intangibles que recibe un cliente al comprar y utilizar los bienes o servicios del minorista (Levy et al., 2014). Una buena propuesta de valor responde a la pregunta 
¿por qué debería comprar este bien o servicio? Hoy en día, una de las mayores fallas en la venta minorista es que muchos minoristas se han concentrado en un solo medio de diferenciación: el precio. También implica la alineación de las competencias centrales de la empresa con las necesidades de la estrategia digital. En esta etapa también se realiza una revisión de las decisiones STP (segmentación, focalización y posicionamiento) para su enfoque en el mundo digital. En relación a la segmentación y focalización, la definición del mercado es clave. Internet ha permitido ampliar el alcance del mercado (Ryan y Jones, 2009). Luego debe establecerse el surtido de productos, el precio y la forma de relacionamiento con los clientes. En relación con el producto, las tecnologías ayudan a crear una nueva propuesta de valor. Si bien es importante buscar nuevas oportunidades para utilizar a la tecnología con el fin de mejorar la oferta, las empresas también deben asegurarse de proteger su posicionamiento competitivo (Chaffe y Ellis-Chadwick, 2016).

La tercera etapa contempla la selección de canales de venta y comunicación para los segmentos de mercado según los objetivos establecidos. La selección de los canales de venta se establece según la naturaleza de los bienes y de los servicios ofrecidos. El desarrollo de una estrategia de marketing digital implica, en muchos casos, el rediseño de los procesos comerciales que realiza la empresa en la actualidad para integrarse con socios, proveedores y distribuidores (Chaffe y Ellis-Chadwick, 2016). También contempla mirar los aspectos positivos de un canal sobre el otro. Las empresas producen una experiencia para el cliente mediante siete elementos de diseño del sitio web: contexto, contenido, comunidad, personalización, comunicación, conexión y comercio. El contexto se refiere al atractivo estético de un sitio web y a la apariencia e impresión de funcionalidad que se reflejan en el diseño y distribución del sitio. Un sitio web orientado a la funcionalidad se centra, en gran medida, en el ofrecimiento de la empresa que podrían ser productos, servicios o información. El contenido abarca toda la información digital incluida en un sitio web e incluye la forma de presentación: texto, video, audio e imágenes. La calidad del contenido y la presentación, junto con las dimensiones del contexto, se combinan para hacer atractivo el sitio web y constituyen la plataforma de los restantes cinco elementos de diseño. La personalización del sitio es su capacidad para modificarse de acuerdo con cada usuario o para que cada persona lo adapte. El elemento de conexión en el diseño del sitio web es la red de vínculos formales entre el sitio de una empresa y otros. Estos vínculos se incorporan al mismo; aparecen como palabras, imágenes o gráficos resaltados y permiten al usuario visitar sin ningún esfuerzo otros sitios con solo hacer clic con el ratón. La comunicación se refiere al diálogo que se establece entre el sitio y sus usuarios. Los consumidores, en particular quienes se registran en un sitio, esperan que la comunicación sea interactiva, individualizada y en tiempo real. Además, un creciente número de páginas digitales de empresas promueve las comunicaciones entre los usuarios, desde el sitio de la empresa, para crear comunidades virtuales o tan solo comunidad. Este elemento de diseño adquiere 
cada vez mayor popularidad porque se ha demostrado que mejora la experiencia del cliente y crea relaciones favorables entre comprador y vendedor. El séptimo elemento de diseño es el comercio y refiere a la capacidad de un sitio web de realizar transacciones de ventas de productos y servicios. Las transacciones en línea son rápidas y sencillas en los sitios bien diseñados (Kerin et al., 2014). El programa de comunicación detalla los pasos para crear una estrategia de comunicación general y ayuda a crear un plan de implementación para desarrollar el mensaje de comunicación. Finalmente debe realizarse una asignación presupuestaria para la combinación de los canales.

La cuarta etapa consiste en la implementación de las operaciones digitales e implica la planificación, implementación, monitoreo y optimización real de las campañas. Las actividades claves incluyen la planificación de las campañas antes de que comiencen y alinean los recursos, las herramientas y las actividades. Comienza con los objetivos de la campaña que incluirían el desarrollo de un resumen de alto nivel del producto, el tipo de segmentos a los que se dirigirá, el mensaje dirigido, las fechas generales de publicación (tiempo de ejecución) y el resultado tangible final deseado en términos de respuesta: tarifas, visitas al sitio web/clientes potenciales o conversiones. Luego debe trabajarse sobre las operaciones reales para configurar la campaña en múltiples canales y se comienza con el desarrollo de un calendario de campaña y su aprobación interna. La ejecución de la campaña involucra todas las actividades realizadas desde el momento de su lanzamiento. Finalmente, culmina con el monitoreo y seguimiento de campañas durante la implementación a partir de puntos de datos diarios y semanales, y métricas extensas para que todos los datos capturados puedan analizarse y monitorearse de manera efectiva.

En la última etapa se realizan informes y un panel de análisis de las métricas con el fin de refinar las estrategias actuales para futuras actividades de marketing digital basadas en la respuesta del cliente y en los datos recopilados. La evaluación de la estrategia implementada puede hacerse a través del análisis de las campañas, donde la empresa aprende más sobre sí misma y sus clientes para crear una próxima interacción (Zahay, 2015). Se pude usar la técnica DMAIC: definir, medir, analizar, actuar y mejorar o eliminar para garantizar que las campañas se ejecuten de la manera más eficaz en futuras producciones. La creciente importancia de la medición en el marketing ha sido paralela al aumento de la importancia del marketing digital (Zahay, 2015).

\section{Conclusión}

El artículo desarrolla la evaluación del marketing hasta su conceptualización actual como filosofía de gestión del negocio, colocando en el centro al cliente al desarrollar procesos para crear, comunicar, entregar e intercambiar ofertas. En este proceso se encuentran involucrados los clientes, los socios y la sociedad en general. El concepto de marketing evolucionó en el tiempo desde la orientación hacia la producción, luego al producto, a la venta y, por último, al marketing. En 
esta evolución, además, se privilegian las relaciones fruto de la incorporación de la tecnología y los medios electrónicos. Los principios de marketing, basados en conceptos sociales, conductuales y económicos, han estado presentes durante muchos años. La segmentación, la selección del mercado y el posicionamiento son decisiones estratégicas que se usan en marketing desde hace décadas.

Luego se analizaron las características del comercio minorista y los cambios a partir de la transformación digital. El negocio minorista tradicional define el formato de negocio a partir de la combinación de decisiones como el mercado objetivo y la ubicación, el surtido, el precio, la comunicación, el formato de atención, el servicio al cliente, la ambientación, el horario y día de prestación y la administración del negocio. En los formatos online, algunas de las variables preponderantes en los negocios offline dejan de tener tanta preponderancia como la localización, las instalaciones, la ambientación, el horario y día de apertura. Las decisiones estratégicas claves para el marketing digital son las mismas que las decisiones estratégicas para el marketing tradicional. Ambas implican seleccionar grupos de cliente objetivo y especificar cómo entregar valor a estos grupos. La pregunta que subyace entonces es: ¿el marketing digital minorista es un nuevo marketing? No es un nuevo marketing, es el mismo, pero se implementa a través de nuevas herramientas. El marketing tradicional, por definición, incluye plataformas y técnicas que se utilizaron como herramientas de comunicación unidireccional en lugar de interactivas, por lo que la tecnología le ha permitido explorar y profundizar aún más estas alternativas.

En tercer lugar, se realizó un análisis de las influencias del comercio minorista a partir de la transformación digital, lo que generó un profundo replanteo en las combinaciones minoristas. La estrategia de marketing digital es principalmente una estrategia de marketing de canal que define cómo una empresa establece objetivos y desarrolla una propuesta de valor diferencial y comunicaciones específicas para un cliente. Como consecuencia de la era digital, proliferaron los canales de venta (las empresas combinan canales físicos y digitales), se redujeron los intermediarios (algunos fabricantes acceden a los clientes a través de formatos online), aparecieron nuevas formas de venta (con intermediarios entre pares, que hacen el vínculo entre ellos) y nuevos canales de comunicación con los clientes (principalmente con la proliferación de las redes sociales). Por otra parte, las empresas no pueden controlar el mensaje (los clientes difunden, comentan y se comunican entre ellos), tienen mayor información disponible (dado por los canales de comunicación y por la interacción entre los clientes), se establecen nuevas formas para fijar precios (en función del cliente, la oferta y la demanda) y la interacción con los clientes es mayor. Los clientes modificaron sus preferencias (la localización ya no es tan importante, las garantías por cambio de productos empieza a ser más valorada), se promueve la respuesta personalizada en función de sus gustos, se otorga mayor importancia a la colaboración en la toma de decisiones y la interacción con la empresa se puede producir en todo momento y lugar. 
Finalmente se presentaron las fases del proceso de marketing digital para un minorista. En este caso comprende las decisiones sobre cómo usar el canal para respaldar la estrategia de marketing existente, cómo explotar sus fortalezas y gestionar sus debilidades, y usarlo junto con otros canales como parte de una estrategia de marketing multicanal. La estrategia de marketing multicanal define cómo los diferentes canales deben integrarse y apoyarse entre sí en términos de desarrollo de propuestas y comunicaciones, en función de sus méritos relativos para el cliente y para la empresa.

Uno de los puntos centrales es el cambio del comportamiento del cliente en tránsito de los modelos tradicionales a digitales. En este sentido, es de interés explorar los principales atributos, beneficios, preferencias, temores y mecanismos que tienen los clientes al realizar compras en canales digitales. El principal aporte puede ser realizar una radiografía del cliente en formato de negocios digitales según el sector comercial pertinente.

\section{Referencias bibliográficas}

Alonso Coto, M. (2008). El plan de marketing digital. Blended marketing como integración de acciones on $y$ offline. Prentice Hall.

Asociación Americana de Marketing. (2020). The definition of marketing. https://www.ama. org/the-definition-of- marketing-what-is- marketing/

Avery, J.; Fournier, S. y Wittenbraker, J. (2014). Unlock the mysteries of your customer relationships. Are you connecting with consumers the way they want you to? Harvard Business Review, 92(7/8), pp. 72-81.

Barker, M.; Barker, D.; Bormann, N. y Neher, K. (2013). Marketing para medios sociales. Un planteamiento estratégico. Cengage Learning.

Berman, B. y Evans, J. (2013). Retail management. A Strategic Approach (12ª ed.). Pearson.

Brinker, S. y McLellan, L. (2014). The rise of the chief marketing technologist. It has become central to marketing, and many companies are creating hybrid executives who straddle the two functions. Harvard Business Review, pp. 82-85.

Charlesworth, A. (2018). Digital marketing. A practical Approach. Routledge.

Chaffey, D. y Ellis-Chadwick, F. (2016). Digital marketing (6 ed.). Pearson Education Limited.

Chaffey, D. y Smith, P. R. (2017). Digital marketing excellence planning, optimizing and integrating online marketing. Routledge.

David, F. (2003). Conceptos de administración estratégica. Prentice Hall.

De Swaan Arons, M.; van den Driest, F. y Weed, K. (2014). The ultimate marketing machine. Harvard Business Review, 59, pp. 54-63.

Edelman, D. (2010). El branding en la era digital. Usted gasta su dinero donde no debe. Harvard Business School, 88(11), pp. 44-51.

Ferrell, O. y Hartline, M. (2018). Estrategia de marketing. Cengage Learning Editores.

180 | Ediciones SAIJ < Ministerio de Justicia y Derechos Humanos de la Nación 
Goworek, H. y McGoldrick, P. (2015). Retail marketing management, principles and practice. Pearson Electronic.

Joshi, A. y Giménez, E. (2014). Marketing impulsado por decisiones. Los buenos procesos de decisión rompen los silos y mejoran el rendimiento. Harvard Business Review, pp. 64-71

Kerin, R.; Harttley, S. y Rudelius, W. (2014). Marketing. Mc Graw Hill.

Kotler, P. y Armstrong, G. (2013). Fundamentos de marketing. Pearson Education.

Kotler, P., Kartajaya, H. y Setiawan, I. (2017). Marketing 4.0 moving from traditional to digital. John Wiley y Sons.

Kotler, P. y Stigliano, G. (2020). Retail 4.0. 10 Reglas para la era digital. LID Editorial.

Lamb, C.; Hair, J. F. y McDaniel, C. (2011). Marketing, Cengage Learning.

Levy, M.; Weitz, B. y Grewal, D. (2014). Retailing management (9a ed). McGraw Hill.

Mintzberg, H.; Quinn, J. y Voyer, J. (1997). El proceso estratégico, conceptos, contextos y casos. Prentice Hall.

Roberts, J. H. (2005). Defensive marketing. How a strong incumbent can protect its position. Harvard Business Review, 83(11), pp. 150-2, 154, 156-7 passim.

Rust, R.; Moorman, C. y Bhalla, G. (2010). Rethinking marketing. Harvard Business Review, 88(1/2), pp. 94-101.

Ryan, D. y Jones, C. (2009). Understanding digital marketing: marketing strategies for engaging the digital. Kogan Page.

Singh Bhatia, P. (2019). Fundamentals of digital marketing (2 $2^{\mathrm{a}}$ ed.). Pearson.

Stanton, W. J.; Etzel, M. J. y Walker, B. (2007). Fundamentos de marketing. Mc Graw Hill.

Zahay, D. (2015). Digital marketing management. Business Expert Press. 